\title{
L'abbaye Saint-Pierre de Mozac (Puy-de-Dôme) : prospection par géoradar
}

Franck Donnadieu, Philippe Labazuy, Denis Hénault, Marine Beziaud et Antoine Coperey

\section{(2) OpenEdition}

Journals

\section{Édition électronique}

URL : https://journals.openedition.org/cem/13499

DOI : $10.4000 /$ cem. 13499

ISSN : 1954-3093

Éditeur

Centre d'études médiévales Saint-Germain d'Auxerre

Référence électronique

Franck Donnadieu, Philippe Labazuy, Denis Hénault, Marine Beziaud et Antoine Coperey, « L'abbaye Saint-Pierre de Mozac (Puy-de-Dôme) : prospection par géoradar », Bulletin du centre d'études médiévales d'Auxerre / BUCEMA [En ligne], 18.2 | 2014, mis en ligne le 19 décembre 2014, consulté le 22 septembre 2022. URL : http://journals.openedition.org/cem/13499 ; DOI : https://doi.org/10.4000/ cem. 13499

Ce document a été généré automatiquement le 22 septembre 2022.

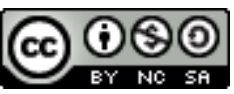

Creative Commons - Attribution - Pas d'Utilisation Commerciale - Partage dans les Mêmes Conditions 4.0 International - CC BY-NC-SA 4.0

https://creativecommons.org/licenses/by-nc-sa/4.0/ 


\title{
L'abbaye Saint-Pierre de Mozac (Puy-de-Dôme) : prospection par géoradar
}

\author{
Franck Donnadieu, Philippe Labazuy, Denis Hénault, Marine Beziaud et \\ Antoine Coperey
}

1 Implanté à $10 \mathrm{~km}$ au nord de ClermontFerrand, le site de Mozac abritait au Moyen Âge l'un des plus anciens et des plus importants monastères d'Auvergne. Fondée sous le règne du roi Thierry III (673-691), l'abbaye n'est mentionnée de façon assurée qu'à partir du IX ${ }^{\mathrm{e}}$ siècle, puis en 1095 lors de son rattachement à Cluny ${ }^{1}$. Conservant les reliques de saint Austremoine, premier évêque présumé de Clermont, l'abbaye fut plusieurs fois reconstruite.

2 Des études de bâti programmées suivies

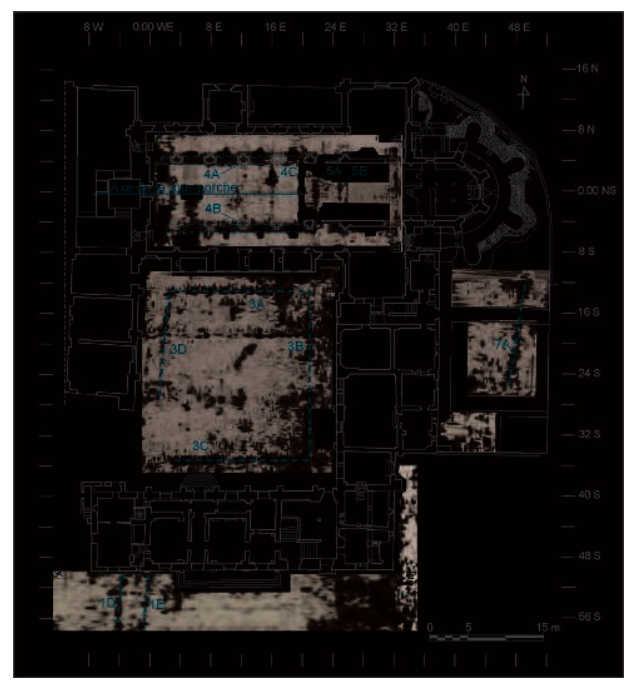
par le Service régional de l'archéologie d'Auvergne ont été menées sur l'abbatiale de 2007 à 2011, puis sur les anciens bâtiments monastiques en 2012 et $2013^{2}$. Ces études ont permis de reconnaître douze phases de construction et/ou de modification entre le $\mathrm{X}^{\mathrm{e}}$ et le $\mathrm{XVIII}^{\mathrm{e}}$ siècle. Une campagne de prospection géophysique, sur l'ensemble du site monastique ${ }^{3}$, a également été conduite par une équipe rattachée à l'université Blaise Pascal de Clermont-Ferrand ${ }^{4}$, durant les mois de mai et juin 2013, en lien avec la mairie de Mozac.

3 Les investigations ont porté sur plusieurs secteurs comprenant l'abbatiale, le cloître, une parcelle située au sud et à l'est de l'ancien réfectoire et trois parcelles situées à l'est du presbytère (fig. 1), soit une surface totale prospectée de $1810 \mathrm{~m}^{2}$ environ. 
4 Si les églises romanes et gothiques en Auvergne ont fait l'objet de nombreuses publications, en revanche l'aspect des cloîtres et des bâtiments monastiques demeure encore mal connu et très peu étudié pour cette région. Les objectifs de la prospection à Mozac étaient de pouvoir préciser l'emplacement des galeries du cloître et de circonscrire l'implantation d'une ancienne salle du monastère roman, raccourcie lors de la reconstruction au $\mathrm{XVII}^{\mathrm{e}}$ siècle de l'aile sud contenant le réfectoire. Une autre attente majeure concernait les vestiges d'une nef préromane s'articulant avec l'ancienne tour-porche occidentale.

\section{Instruments et contraintes ${ }^{5}$}

5 Un radar d'auscultation (géoradar SIR-3000 de GSSI) équipé d'une antenne blindée de $400 \mathrm{MHz}$ a été utilisé pour prospecter l'ensemble des secteurs cibles de l'abbaye, avec un maillage de $50 \mathrm{~cm}$ de côté. Un maillage plus serré $(10 \mathrm{ou} 20 \mathrm{~cm})$ a été effectué en recouvrement dans certaines zones d'intérêt. Une antenne blindée de $200 \mathrm{MHz}$ a été employée suivant un maillage de $25 \mathrm{~cm}$ afin de sonder plus profondément - et avec un meilleur rapport signal sur bruit dans la tranche 2-3 m - une zone de 5 x $13 \mathrm{~m}$ au sudest du bâtiment sud, dans l'optique de fouilles futures.

Deux autres instruments ont été testés sur certaines parcelles - conductivimètre trifréquence EMP-400 de GSSI, et gradiomètre GSM-19 de GEM Systems -, mais la présence de nombreux objets métalliques a considérablement limité les capacités d'investigation et les résultats ne sont pas présentés ici.

\section{Résultats}

\section{Abbatiale Saint-Pierre}

Dans l'ancienne abbatiale le radar-sol a permis d'identifier au moins cinq structures situées entre $0,70 \mathrm{~m}$ et $1,90 \mathrm{~m}$ de profondeur. Les principales structures recherchées étaient les vestiges des murs d'une ancienne nef s'articulant avec la tour-porche préromane. Découverts lors de sondages menés en 1995 entre les piles de la nef romane ${ }^{6}$, ces murs orientés ouest-est ne furent identifiés qu'en 2009 à l'issue de l'étude archéologique du bâti de la tour-porche ${ }^{7}$. Apparaissant à une altitude moyenne de 0,60 à - 0,70 m, ces maçonneries n'avaient pas été relevées sur toute leur longueur et nous ignorions la profondeur jusqu'à laquelle elles sont conservées.

8 La prospection géophysique a permis de reconnaître très nettement des structures linéaires ( $4 \mathrm{~A}$ et $4 \mathrm{~B})$, situées à peu près dans l'alignement des piles de la nef romane, et commençant à apparaître à $0,70 \mathrm{~m}$ jusqu'à $1,90 \mathrm{~m}$ de profondeur (fig. 1). 
Fig. 1 - Images des réflexions radar à différentes profondeurs : - 1,80 m pour les secteurs 1 et 2 au sud du bâtiment sud (sauf zone restreinte au sud-est à - $2 \mathrm{~m}$ ) ; - 1,50 m pour le secteur 3 (cloître) ; $1,60 \mathrm{~m}$ pour le secteur 4 (nef et bas-côtés) ; $1,40 \mathrm{~m}$ pour le secteur 5 (dernière travée de la nef et croisée du transept) ; - 1,60 m pour les secteurs 6 et 7 situés à l'est du presbytère (bâtiment est) ; plan D. Hénault, 2012, et images radar F. Donnadieu, P. Labazuy, A. Coperey et M. Beziaud, 2013.

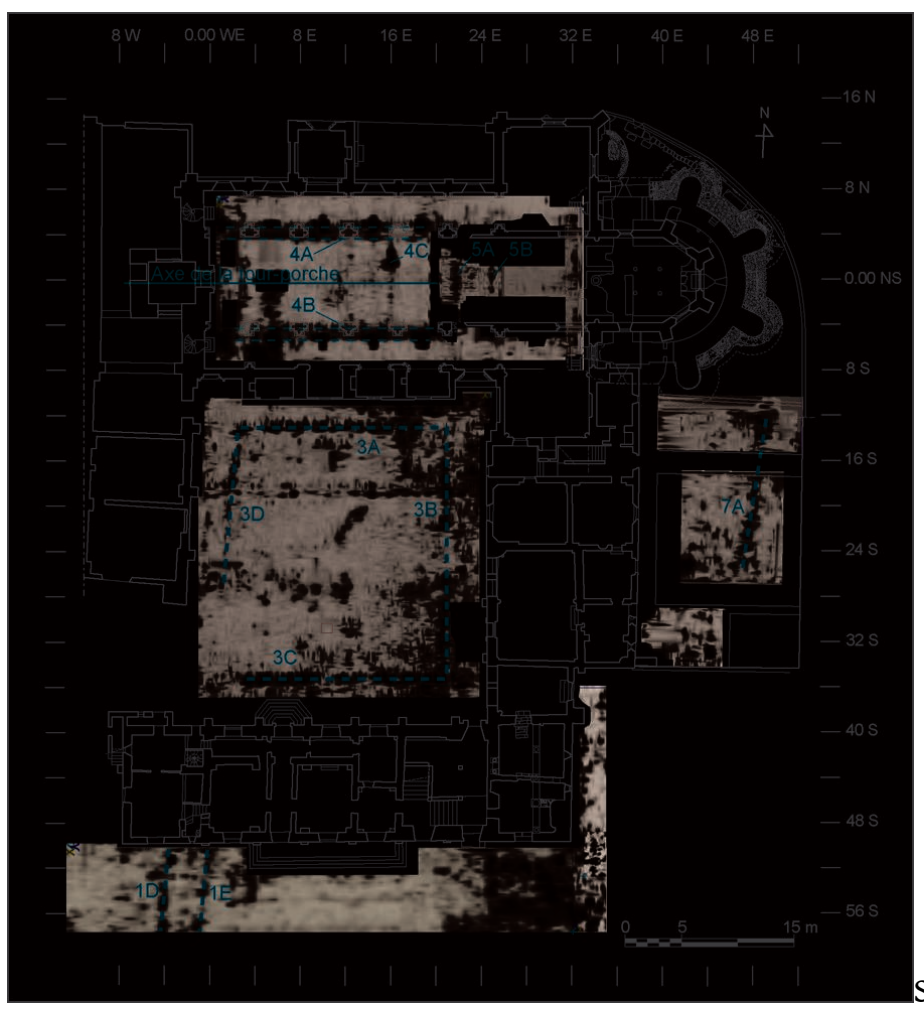

S'étendant sur une vingtaine de mètres en longueur depuis le revers de la façade ouest, elles s'interrompent à l'est dans la tranche 18-22 WE au niveau des cinquièmes piles nord et sud de la nef actuelle. Par ailleurs, ces structures ne sont pas alignées toutes les deux sur l'axe de la nef romane. Ces deux alignements se trouvent au contraire à égale distance de part et d'autre de l'axe médian de la tour-porche préromane. Le recoupement entre l'ensemble des données archéologiques disponibles et celles issues de la prospection géophysique ne laisse aucun doute sur l'identification de ces structures, qui correspondent aux vestiges des murs d'une nef unique s'articulant avec la tour-porche occidentale. Notons que les structures repérées lors des sondages et de la prospection radar sont approximativement conservées sur un peu plus d'un mètre d'élévation - avec sans doute le niveau de fondation. Comme cela avait été pressenti, les murs gouttereaux de l'ancienne nef furent donc partiellement arasés et le niveau de sol surhaussé, afin de consolider les fondations destinées à supporter les piles de la nef romane ${ }^{8}$.

9 Par ailleurs, l'absence totale de signaux dans les bas-côtés ainsi que dans la nef hormis les structures 4D-F et 5A-B décrites ci-dessous - pourrait démontrer qu'il n'y a eu aucune tripartition de la nef préromane ni aucun ajout de bas-côtés au XI ${ }^{\mathrm{e}}$ siècle. La nef préromane a peut-être été conservée jusqu'à la construction de la grande abbatiale romane du XII siècle. La question alors soulevée est celle de l'existence d'une abside plus à l'est. Les proportions de la nef préromane permettent de situer une éventuelle abside sous la dernière travée de la nef romane. La prospection radar a été compliquée par la 
présence de mobilier dans ce secteur et d'un treillis métallique armant l'estrade de l'autel. Malgré le seuil de bruit élevé, deux structures linéaires (5A et 5B) orientées nord-sud se dessinent entre 1,10 et $1,70 \mathrm{~m}$ de profondeur. Le réflecteur $5 \mathrm{~A}$, d'épaisseur submétrique et mesurant au moins $3 \mathrm{~m}$ en longueur, pourrait éventuellement représenter les traces du mur de chaînage de l'arc de tête d'une abside, mais seule l'archéologie permettrait de le confirmer. Le réflecteur 5B, $3 \mathrm{~m}$ plus à l'est, est aminci aux deux tiers de sa longueur $(2,40 \mathrm{~m})$ et possède des contours plus irréguliers, qui pourraient être compatibles avec le tracé de l'abside attendue. Mais son interprétation reste incertaine d'autant qu'il pourrait aussi s'agir de deux objets distincts.

11 L'étude géophysique a par ailleurs révélé l'existence d'une barrière liturgique (4D) repérable entre 0,70 et $1,30 \mathrm{~m}$ de profondeur, d'orientation nord-sud et située à l'aplomb des colonnes engagées occidentales des quatrièmes piles nord et sud de la nef (fig. 2). Si l'ensemble des données disponibles ne laisse aucun doute sur l'identification de cette structure, la période à laquelle elle peut être rattachée demeure incertaine ${ }^{9}$. Un autre objet (4C, fig. 1), difficilement interprétable (environ $1 \times 2 \mathrm{~m}$ ), apparait sous la partie nord de cette structure entre 1,50 et 1,90 $\mathrm{m}$ de profondeur.

12 Notons enfin la présence sur le relevé de deux anomalies (4E et $4 \mathrm{~F}$ ) de forme quadrangulaire, légèrement excentrées vers le nord et situées, l'une, entre les troisièmes piles nord et sud de la nef (fig. 2), l'autre, au revers de la structure D, dans la quatrième travée. La position, les dimensions, l'épaisseur - entre 30 et $40 \mathrm{~cm}$ de hauteur - et la forme de ces structures permettent de proposer prudemment l'existence de caveaux.

Fig. 2 - Réflexions radar à - 0,90 $\mathrm{m}$ dans les secteurs 4 et 5 (nef, bas-côtés et croisée du transept) ; plan D. Hénault, 2012, et images radar F. Donnadieu, P. Labazuy, A. Coperey et M. Beziaud, 2013.

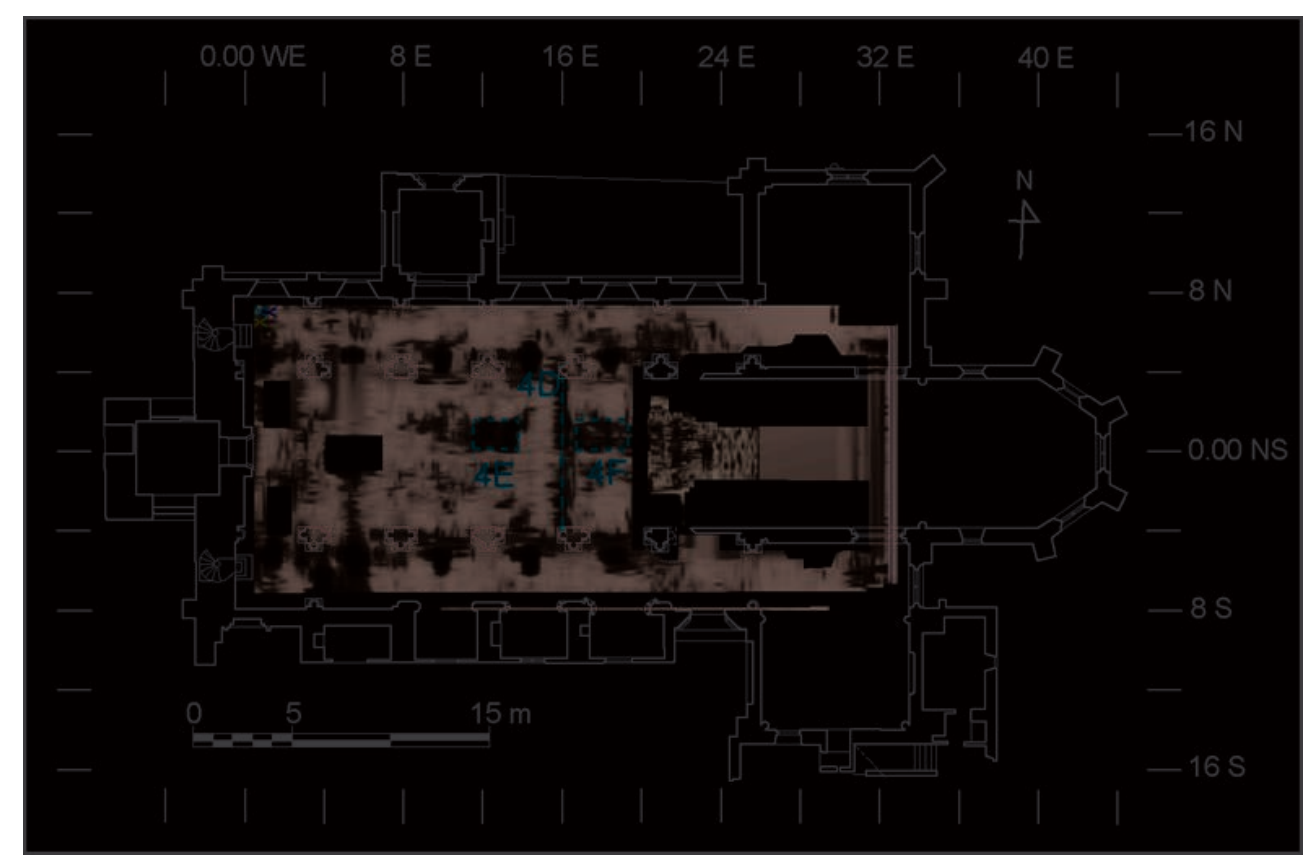

\section{Cloître}

Les galeries subsistantes du cloître acquises après la Révolution par des particuliers furent progressivement démolies au XIX ${ }^{\mathrm{e}}$ siècle. Plusieurs indices conservés en élévation 
ont permis de définir approximativement l'implantation de plusieurs galeries des cloîtres successifs, dont l'extension et le plan évoluèrent sensiblement au gré des nombreuses reconstructions qui affectèrent l'abbaye.

La cartographie géophysique montre, à 1,50 $\mathrm{m}$ de profondeur, quatre bandes linéaires peu épaisses (environ $0,70 \mathrm{~m}$ ), situées chacune à un peu moins de $4 \mathrm{~m}$ des façades actuelles et dessinant un quadrilatère non fermé à l'ouest (fig. 1). Correspondant aux quatre galeries de l'ancien cloître (3A-D), ces structures apparaissent à une altitude s'accordant parfaitement avec les cotes altimétriques relevées dans la salle romane, le niveau de sol actuel de l'ancien cloître étant très surhaussé ${ }^{10}$. En outre, la largeur des galeries est et nord est clairement déterminée par celle des travées de l'abbatiale romane. Par ailleurs, la carte géophysique semble confirmer l'existence de niveaux superposés (différentes périodes), puisque des parties des galeries nord et sud apparaissent dès $1 \mathrm{~m}$ de profondeur. À l'ouest, le tracé reprenant l'orientation légèrement désaxée NNE-SSW de l'ancien cuvage est incomplet et apparait moins nettement, peut-être en raison de l'humidité du sol, plus importante dans cette zone.

\section{Parcelles sud et sud-est}

Les études archéologiques inédites menées en 2012 dans une ancienne salle du monastère roman ont permis de situer approximativement les murs est et sud démolis au $\mathrm{XVII}^{\mathrm{e}}$ siècle lors de la reconstruction de l'aile méridionale contenant l'ancien réfectoire ${ }^{11}$. L'objectif de l'étude géophysique était de préciser l'emplacement exact et l'altitude des vestiges de ces maçonneries. L'étude menée avec l'antenne $200 \mathrm{MHz}$ a permis de faire apparaître très nettement le plan de la travée manquante de l'ancienne salle romane. Ainsi la cartographie montre, entre $1,80 \mathrm{~m}$ et $2 \mathrm{~m}$ de profondeur, trois structures linéaires ( $1 \mathrm{~A}-\mathrm{C}$, fig. 3 ) épaisses d'environ $1 \mathrm{~m}$ et orientées nord-sud, pour deux d'entre elles, et ouest-est, pour la troisième. 
Fig. 3 - Réflexions radar à - $2 \mathrm{~m}$ au sud-est du secteur 1 (bâtiment sud) obtenues avec une antenne $400 \mathrm{MHz}$ suivant des profils croisés tous les $50 \mathrm{~cm}$ (partie est), et améliorées au sud avec une antenne $200 \mathrm{MHz}$ et des profils croisés à $25 \mathrm{~cm}$, révélant les structures archéologiques profondes (plan D. Hénault, 2012, et images radar F. Donnadieu, P. Labazuy, A. Coperey et M. Beziaud, 2013).

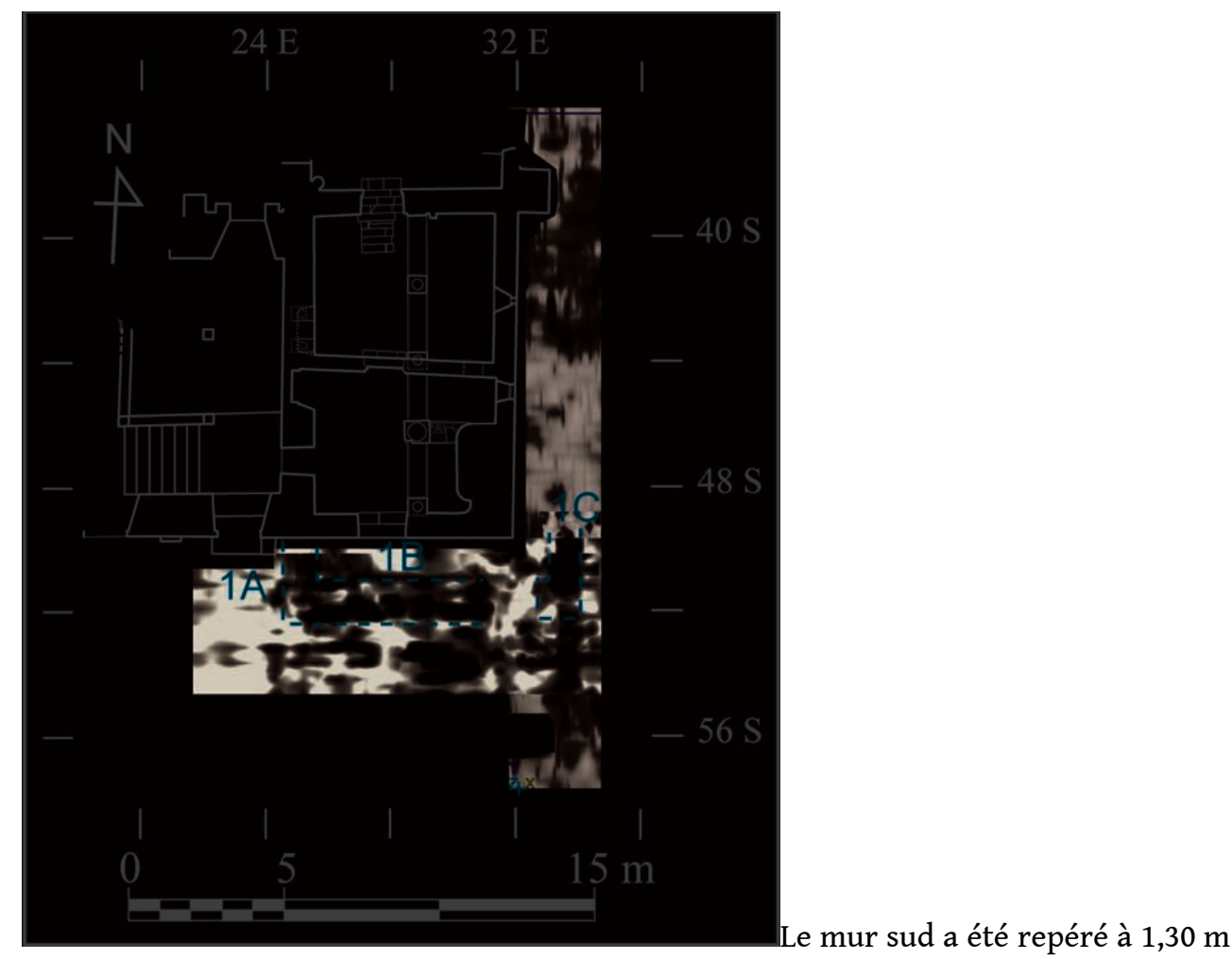
de distance de la façade actuelle, soit exactement les dimensions restituables en tenant compte de la moyenne entraxe des colonnes encore en place dans la salle romane ${ }^{12}$.

$\mathrm{Au}$ sud de ces structures apparaissent également, aux mêmes profondeurs, plusieurs tracés orthogonaux, qui pourraient correspondre à une autre salle (?) d'époque indéterminée, se prolongeant au-delà de la zone prospectée.

17 Par ailleurs, dans la partie occidentale du secteur sud, deux structures linéaires (1D et $1 \mathrm{E}$, fig. 1) sub-parallèles orientées nord-sud sont décelables à partir de 1,20 $\mathrm{m}$ jusqu'à $1,90 \mathrm{~m}$ de profondeur. Difficilement interprétables en l'état, elles s'étendent sur $8 \mathrm{~m}$ de longueur et semblent continuer plus au sud, au-delà de la zone prospectée.

\section{Parcelles est}

L'étude archéologique et archivistique menée en 2012 et portant sur les bâtiments conventuels a soulevé la question d'une possible chapelle mariale, située à proximité de la salle capitulaire ${ }^{13}$. L'objectif de la prospection dans ce secteur était donc de repérer d'éventuels vestiges pouvant appartenir à ce type de chapelle, connue et attestée dans plusieurs grands sites clunisiens ${ }^{14}$. À l'issue de l'étude par géoradar à $400 \mathrm{MHz}$, aucune structure de cette nature n'a pu être relevée dans la cour et les jardins du presbytère ${ }^{15}$.

\section{Bilan de la prospection}

19 La prospection radar et les études menées pendant deux ans à Mozac sont venues renouveler très largement nos connaissances sur l'occupation et l'évolution 
architecturale du site. Les résultats sont d'abord particulièrement significatifs pour l'abbatiale, dont la nef actuelle dissimule les vestiges d'une nef unique charpentée et peut-être aussi d'une abside, contemporaines de la tour-porche préromane conservée en élévation, ainsi que la façade occidentale du chœur monastique - fondation et/ou élévation d'une barrière liturgique. De même, les prospections ont également été concluantes dans le cloître dont les galeries ont pu être reconnues.

Par ailleurs, les études portant sur une ancienne salle romane, partiellement conservée en élévation et dont l'usage demeure incertain (salle commune, chauffoir ?), autorisent désormais des comparaisons avec d'autres salles contemporaines appartenant à des sites tels que Billom, Saint-Saturnin, Royat ou bien encore Saint-Dier-d'Auvergne.

Si les investigations n'ont rien donné sur les parcelles situées à l'est du presbytère, l'existence d'un dispositif clunisien, de type chapelle mariale s'articulant avec le chapitre, demeure encore envisageable à Mozac et de futurs sondages pourraient révéler quelques surprises. De plus, les différentes structures potentielles repérées sur l'ensemble du site pourront orienter de futurs travaux de fouilles ou des sondages, afin de pouvoir préciser les hypothèses avancées plus haut et dater certaines maçonneries.

Si l'aspect et la topographie des monastères ont fait l'objet de synthèses dans différentes régions ${ }^{16}$, ce sujet demeure encore mal connu et peu étudié pour l'Auvergne. La prospection et les études achevées ou en cours à Mozac et sur d'autres sites - Aurillac, Souvigny, Sauxillanges - apportent donc des éléments nouveaux pour tenter de remédier à cette situation. De nouvelles orientations de recherche peuvent alors être définies, en envisageant à la fois des études thématiques et monographiques pour tenter de mieux comprendre l'aspect et l'organisation spatiale des différents monastères en Auvergne.

Reçu : 14 janvier 2014 - Accepté : 2 décembre 2014

\section{NOTES}

1. A. DIERKENS, "Une abbaye médiévale face à son passé. Saint-Pierre de Mozac du $\mathrm{IX}^{\mathrm{e}} \mathrm{au}$ $\mathrm{XII}^{\mathrm{e}}$ siècle ", in Écrire son histoire. Les communautés régulières face à leur passé, Saint-Étienne, 2005 p. 71-105.

2. Sur l'abbatiale: D. HÉNAULT, Rapport de synthèse sur les études d'élévations de l'église Saint-Pierre de Mozac (Puy-de-Dôme) menées de 2007 à 2011, dernière campagne d'étude (15 septembre-15 octobre 2011), SRA/DRAC Auvergne, 2012 ; ID., "Mozac (Puy-de-Dôme). Église Saint-Pierre", Archéologie médiévale, 42 (2012), p. 247 ; sur les bâtiments conventuels : ID. (coord.), L'ancienne abbaye SaintPierre de Mozac, rapport final de synthèse sur les études archéologiques menées en 2012 et 2013. Dernière campagne d'étude (avril-décembre 2013), SRA/DRAC Auvergne, 2014.

3. Sur les résultats de la prospection: F. DonnAdieu, P. LABAZuY, D. HÉNAULt, M. BEZIAud et A. COPEREY, Abbaye Saint-Pierre à Mozac (63). Rapport d'étude géophysique, université Blaise Pascal, Clermont-Ferrand, 2013 ; EID., Abbaye Saint-Pierre à Mozac (63). Addendum au rapport géophysique : prospection géoradar à $200 \mathrm{MHz}$, université Blaise Pascal, Clermont-Ferrand, 2014. 
4. L'équipe était composée de Franck Donnadieu et Philippe Labazuy, physiciens adjoints Observatoire de physique du globe de Clermont-Ferrand, laboratoire Magmas et Volcans (UMR 6524 CNRS-IRD) -, Antoine Coperey et Marine Beziaud, étudiants en master Sciences de la terre à l'université Blaise Pascal de Clermont-Ferrand. La coordination était assurée par Denis Hénault, archéologue du bâti, responsable des études sur le site de Mozac. L'étude fut conduite du 29 avril au 24 mai 2013 et complétée durant les mois de juin et septembre sur des secteurs particuliers.

5. Sur le principe du radar utilisé pour l'archéologie : L. CONYERS, Ground-penetrating radar for archaeology, 2004.

6. P. ARNAUD, Mozac (Puy-de-Dôme) : abbatiale Saint-Pierre. Rapport de surveillance archéologique de travaux, Clermont-Ferrand, DRAC/SRA Auvergne, 1995.

7. D. HÉNAULT, "Saint-Pierre de Mozac, la tour-porche et l'abbatiale du haut Moyen Âge ", Bulletin historique et scientifique de l'Auvergne, 110 (2009), p. 47-82 ; ID., Mozac (Puy-de-Dôme), étude archéologique du bâti de la tour-porche de l'église Saint-Pierre. Rapport d'intervention, ClermontFerrand, SRA/DRAC Auvergne, 2010, p. 49-52.

8. D. HÉNAULT, « Saint-Pierre de Mozac, la tour-porche et l'abbatiale du haut Moyen Âge... », ibid., p. 79.

9. Compte tenu des données disponibles, seules deux interprétations peuvent être retenues quant à la période à laquelle peut appartenir cette structure. La première solution serait celle de l'emplacement de l'ancien jubé édifié après 1490 et dont subsiste l'ancien portail central remonté au XVIII ${ }^{\mathrm{e}}$ siècle à l'entrée ouest de la nef. Néanmoins, l'ensemble des données archéologiques conservées en élévation plaiderait plutôt en faveur d'un emplacement du jubé situé plus à l'est, entre les cinquièmes piles nord et sud de la nef. La seconde interprétation possible est celle d'une barrière liturgique romane. L'altitude de la structure repérée par rapport au niveau de sol connu de la nef romane - 354.80 à $355,00 \mathrm{~m} \mathrm{NGF}$, en moyenne - s'accorderait tout à fait avec cette solution, de même que l'emprise et le positionnement du chœur liturgique dans ce cas de figure. En revanche, l'absence de tout vestige connu de barrière de chœur romane à Mozac fragilise cette hypothèse. La question reste donc ouverte.

10. Les vestiges repérés apparaissent à environ $353,80 \mathrm{~m}$ NGF tandis que le sol roman de la salle se situe à 353,65 m NGF. Au XII ${ }^{\mathrm{e}}$ siècle, le sol du cloître et celui du rez-de-chaussée des bâtiments situés autour étaient beaucoup plus bas qu'aujourd'hui. Le sol du cloître a été surélevé successivement aux XVI ${ }^{\mathrm{e}}, \mathrm{XVII}^{\mathrm{e}}$ et XIX ${ }^{\mathrm{e}}$ siècles.

11. D. HÉNAULT, Rapport sur les études archéologiques du bâti et les sondages menés dans les anciens bâtiments conventuels de l'abbaye de Mozac. Première campagne d'étude, Clermont-Ferrand, SRA/DRAC Auvergne, 2013, p. 19-22.

12. Ainsi la salle romane pourrait mesurer un peu moins de $7 \mathrm{~m}$ de large sur $11,70 \mathrm{~m}$ de longueur dans œuvre.

13. D. HÉNAULT, Rapport sur les études archéologiques du bâti et les sondages menés dans les anciens bâtiments conventuels..., op. cit., p. 24-25, 27 et 34.

14. Autour du cloitre: les chapelles Notre-Dame et les accès au chapitre, éd. Bucema, hors-série $n^{\circ} 6$, 2013 [http://cem.revues.org].

15. Entre $1,50 \mathrm{~m}$ et $1,80 \mathrm{~m}$ de profondeur, une structure linéaire (7A, fig. 1 ) d'épaisseur métrique orientée $\mathrm{N} 15^{\circ}$ a été repérée. Cette structure pourrait atteindre environ $14 \mathrm{~m}$ de longueur du nord au sud dans la tranche 12-28 NS. La position de cette structure, son orientation, sa profondeur et les traces plus ou moins ténues repérées sur la cartographie rendent difficile toute interprétation en l'état. Il pourrait aussi bien s'agir des fondations d'un bâtiment - d'époque et d'usage indéterminés - que de négatifs de structures de nature diverse - annexe, système défensif, etc.

16. C. TREFFORT et P. BRUDY (dir.), Monastères entre Loire et Charente, Rennes, 2013 ; J.-F. REYNAUD, M. PACAUT ( $\dagger$ ) et M. WULLSCHLEGER, Espaces monastiques ruraux en Rhône-Alpes, 2002 (DARA, 23). 
INDEX

Mots-clés : abbaye, prospection radar

Index géographique : France/Mozac

\section{AUTEURS}

FRANCK DONNADIEU

Université Blaise Pascal Clermont II, OPGC et Laboratoire Magmas et Volcans

\section{PHILIPPE LABAZUY}

Université Blaise Pascal Clermont II, OPGC et Laboratoire Magmas et Volcans

\section{DENIS HÉNAULT}

Commune de Mozac et Communauté de communes de Riom

\section{MARINE BEZIAUD}

Université Blaise Pascal Clermont II, OPGC et Laboratoire Magmas et Volcans

\section{ANTOINE COPEREY}

Université Blaise Pascal Clermont II, OPGC et Laboratoire Magmas et Volcans 